\title{
Optical properties of free-standing GaAs semiconductor nanowires and their dependence on the growth direction
}

\author{
P. Redliński* and F. M. Peeters ${ }^{\dagger}$ \\ Departement Fysica, Universiteit Antwerpen, Groenenborgerlaan 171, B-2020 Antwerpen, Belgium \\ (Received 12 July 2007; revised manuscript received 13 October 2007; published 26 February 2008)
}

\begin{abstract}
The electronic states of a free-standing widegap semiconductor quantum nanowire are investigated. We applied the variational technique using the effective mass approximation within the $\mathbf{k} \cdot \mathbf{p}$ model to a thin cylindrical GaAs nanowire. The energy dispersion, the distribution of the spinor components, and the optical absorption spectra are presented. The hole dispersion shows a typical "camel back" structure. The effect of the dielectric mismatch between the wire and the surrounding medium and the influence of the growth direction of the wire are examined. We find that the photoluminescence energy of the exciton is larger in the [001] configuration than the [111] configuration.
\end{abstract}

DOI: 10.1103/PhysRevB.77.075329

PACS number(s): 73.21.Hb

\section{INTRODUCTION}

Growing interest in nanosized crystalline semiconductor structures in conjunction with novel growth mechanisms such as the vapor-liquid-solid ${ }^{1}$ technique (VLS) or the molecular beam epitaxy technique ${ }^{2}$ have allowed the synthesis of high-quality nanowires and nanorods of almost every group of semiconductors, including group IV semiconductors [Si (Ref. 3) and Ge] as well as III-V and II-VI compounds $\left[\mathrm{InAs},{ }^{4} \mathrm{GaAs}^{5,6}{ }^{5} \mathrm{InP},{ }^{7}\right.$ and $\mathrm{CdSe}$ (Ref. 8)]. The radius of these nanowires typically ranges from one to a few tens of nanometers, but their length can easily exceed micrometers. Zinc-blende and diamondlike free-standing nanowires mentioned above usually grow along the [001], [110], or [111] crystallographic directions depending on the size of the nanowire and the growth conditions. The energy dispersion also depends on the size, shape, and environment of the nanowires. It turns out that the VLS growth technique is very flexible allowing for growth of, e.g., superlattice structures ${ }^{9}$ and core-shell structures. ${ }^{10}$ In a core-shell nanostructure, one or more shells surround the core material usually with a smaller band gap material. Most VLS nanowires exhibit a large dielectric mismatch with their surroundings resulting in a large correction to the bare Coulomb interaction and therefore this dielectric mismatch effect is very important in the study of their optical properties as was shown previously. ${ }^{11}$

Quantum wires are under active investigation because devices based on them offer important opportunities as building blocks for the next generation of electronic and optoelectronic devices. Nanowires are excellent laboratory systems for probing one-dimensional physics with plenty of opportunities both for experimentalists and theoreticians. ${ }^{12}$ Quantum wire structures have density of states features, which are very useful for laser ${ }^{13}$ applications with possibility of smaller current threshold density than in lasers produced from higher dimensional structures. Furthermore, nanowires are building components for other nanoscale devices such as photodetectors, ${ }^{14}$ light-emitting diodes, field-effect transistors, ${ }^{15}$ etc. A wide range of interesting transport and optical properties are already unveiled, such as, e.g., strong luminescence polarization ${ }^{7}$ and clean Coulomb blockade features at low temperature.
In this paper, we investigate the electronic properties of free-standing nanowires using the $\mathbf{k} \cdot \mathbf{p}$ approach. Our model system is an infinitely long free-standing wire of cylindrical cross section (see Fig. 1). In this scheme, we study quasione-dimensional states, states which are quantized in the lateral direction and propagating along the wire. There are also two-dimensional surface states which are completely neglected in this paper but we refer the reader, for example, to Ref. 16. The effect of the growth direction on the electronic properties will be investigated and therefore we consider two cases: in the first case, the nanowire is grown along the [001] crystallographic direction and in the second case, it is grown along the [111] direction. As an example, we present numerical results for GaAs nanowires for which in bulk we have the two characteristic scales: the bulk Bohr radius $a_{B}$ $\approx 11.5 \mathrm{~nm}$ and the effective three-dimensional Rydberg en$\operatorname{ergy} E_{R y}^{*} \approx 5.0 \mathrm{meV}$. We will study the regime of a thin nanowire with diameter $D=2-12 \mathrm{~nm}$, so $D \leqslant a_{B}$. It implies that quantum confinement effects are very important as will be shown directly by our results.

The paper is organized as follows. In the next section, we describe our theoretical model. In Sec. III, we present results for the one-particle states with emphasis on quantum confinement effects. The one-particle subband structure is a key to the understanding of charge transport in a nanowire. Subsequently, excitonic states are studied where we include the
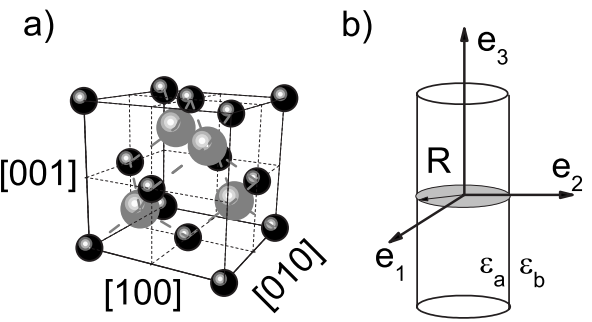

FIG. 1. (a) fcc lattice of zinc blende with crystallographic axes [100], [010], and [001]. (b) Natural system of coordinates $\left(\vec{e}_{1}, \vec{e}_{2}, \vec{e}_{3}\right)$ for a cylindrical wire with radius $R . \epsilon_{a}$ and $\epsilon_{b}$ are dielectric constants inside and outside the nanowire, respectively. For a typical free-standing wire, there is a large dielectric mismatch, i.e., $\epsilon_{a} \gg \epsilon_{b}$. 
dielectric mismatch effect. In the last section, we summarize our main findings.

\section{THEORY}

Here, we present details of the $\mathbf{k} \cdot \mathbf{p}$ model as applied to our quantum system. Our investigation concentrates on direct wide gap semiconductors such as GaAs or CdTe. We neglect the interaction between conduction and valence bands which is small for wide gap compounds. Electrons in the conduction band are modeled by a quadratic Hamiltonian with effective mass $m^{*}$. On the other hand, for the valence band, we include the spin-orbit interaction. We use the exact form of the Kohn-Luttinger Hamiltonian ${ }^{17,18}$ (KL) without making any additional approximations as, for example, the axial or the spherical approximations that are often used in the literature. ${ }^{19}$

We investigated nanowires grown along the [001] or the [111] crystallographic direction. In our calculations, we used the KL with spin quantization along the direction of the wire, ${ }^{20,21}$ i.e., along the [001] or the [111] crystallographic directions. Specifically, according to the notation of Fig. 1, we used two systems of coordinates $\left\{\vec{e}_{1}, \vec{e}_{2}, \vec{e}_{3}\right\}=\{[100],[010],[001]\}$ and $\left\{\vec{e}_{1}, \vec{e}_{2}, \vec{e}_{3}\right\}=\{[11-2]$, $[-110],[111]\}$, respectively. For a free-standing nanowire, it is a good approximation to assume that the electronic charge is totaly localized in the region of the nanowire and is exactly zero outside the wire.

According to the above assumptions, the envelope wave function of the conduction band has the following form:

$$
\Psi_{e l}(\rho, \phi, z, \sigma)=e^{i k_{z} z} \frac{e^{i m \phi}}{\sqrt{2 \pi}} \frac{\sqrt{2}}{R J_{m+1}\left(\alpha_{m l}\right)} J_{m}\left(\alpha_{m l} \frac{\rho}{R}\right) \eta_{\sigma},
$$

where $J_{m}$ is a Bessel function of the first kind and $\alpha_{m l}$ is the $l$ th zero of the corresponding $J_{m}(m=0, \pm 1, \pm 2, \ldots$, $l=0,1,2, \ldots)$ and $R$ is the radius of the wire. We used cylindrical coordinates $(\rho, \phi, z)$. Eigenenergies form a set of subbands numbered by pairs $(m, l)$ and are twofold degenerate with respect to the spin degree of freedom: $\sigma= \pm$. In Eq. (1), the symbol $\eta_{\sigma}$ indicates the spin part of the electron wave function.

For the valence band, for each component $\nu$ of the spinor, we assumed the following trial wave function:

$$
\begin{aligned}
\Psi_{h}(\vec{r}, \nu) & =\Psi_{h}(\rho, \phi, z, \nu) \\
& =e^{i k_{z} z} \sum_{m=-M}^{M} \sum_{l=0}^{L-1} C_{m, l}^{\nu} \frac{e^{i m \phi}}{\sqrt{2 \pi}} \frac{\sqrt{2}}{R J_{m+1}\left(\alpha_{m l}\right)} J_{m}\left(\alpha_{m l} \frac{\rho}{R}\right) .
\end{aligned}
$$

Typically, only a few $m$ 's and $l$ 's are needed to achieve convergence for low energy eigenpairs. Amplitudes $C_{m, l}^{\nu}$ are determined by diagonalizing the KL. The same number of terms in Eq. (2) was used for the [001] and [111] cases.

Having calculated the energy spectra and corresponding wave functions, we then calculated the absorption power using the Fermi golden rule. ${ }^{22}$ Abbreviating $i$ stands for the initial electronic state and $f$ for the final electronic state and the absorption power of photons with energy $E_{p h}=\hbar \omega_{p h}$ reads

$$
P=\frac{\pi e^{2}}{2 \omega_{p h}} \sum_{i, f}\left(f_{i}-f_{f}\right)|\langle f|\vec{p} \cdot \vec{A}| i\rangle|^{2} \delta\left[E_{f}-\left(E_{i}+\hbar \omega_{p h}\right)\right],
$$

where $f_{i / f}$ is the Fermi-Dirac distribution function of initial $(i)$ and final $(f)$ electronic states, respectively. Initial state corresponds to the valence band state $\langle\vec{r} \mid i\rangle$ $=\Sigma_{\mu} \Psi_{h}(\vec{r}, \mu)\langle\vec{r} \mid \mu\rangle$ and the final state corresponds to the conduction band state $\langle\vec{r} \mid f\rangle=\Psi_{e l}(\vec{r})\langle\vec{r} \mid S\rangle$, where $\langle\vec{r} \mid \mu\rangle$ and $\langle\vec{r} \mid S\rangle$ are Bloch states at the $\Gamma$ point of the Brillouin zone. For three different polarizations of light, the integral $I$ $=|\langle f|\vec{p} \cdot \vec{A}| i\rangle|^{2}$ was approximated as follows:

$$
\begin{aligned}
I_{\sigma^{+}} \simeq & \mid \frac{2}{\sqrt{6}}\left\langle\Psi_{e l, \uparrow} \mid \Psi_{h, 3 / 2,-1 / 2}\right\rangle+\frac{2}{\sqrt{2}}\left\langle\Psi_{e l, \downarrow} \mid \Psi_{h, 3 / 2,-3 / 2}\right\rangle \\
& -\left.\frac{2}{\sqrt{3}}\left\langle\Psi_{e l, \uparrow} \mid \Psi_{h, 1 / 2,-1 / 2}\right\rangle\right|^{2}, \\
I_{\sigma^{-}} \simeq & \mid \frac{2}{\sqrt{2}}\left\langle\Psi_{e l, \uparrow} \mid \Psi_{h, 3 / 2,+3 / 2}\right\rangle+\frac{2}{\sqrt{6}}\left\langle\Psi_{e l, \downarrow} \mid \Psi_{h, 3 / 2,+1 / 2}\right\rangle \\
& +\left.\frac{2}{\sqrt{3}}\left\langle\Psi_{e l, \downarrow} \mid \Psi_{h, 1 / 2,+1 / 2}\right\rangle\right|^{2}, \\
I_{\pi} \simeq \mid & \sqrt{\frac{2}{3}}\left\langle\Psi_{e l, \downarrow} \mid \Psi_{h, 3 / 2,-1 / 2}\right\rangle+\left.\sqrt{\frac{2}{3}}\left\langle\Psi_{e l, \uparrow} \mid \Psi_{h, 3 / 2,+1 / 2}\right\rangle\right|^{2} .
\end{aligned}
$$

Equation (3) was integrated numerically for $T=0 \mathrm{~K}$ so we assumed that the valence band is totally full and the conduction band is totally empty and consequently the factor $\left(f_{i}\right.$ $\left.-f_{f}\right)=1$. For $R<6 \mathrm{~nm}$, the separation between the conduction and the valence band states in GaAs is larger than $1.5 \mathrm{eV}$ so we expect that the relation $\left(f_{i}-f_{f}\right)=1$ is also approximately fulfilled even close to room temperature.

We analyzed also the electron-hole bound states (exciton) in a medium with dielectric mismatch. The Hamiltonian of the exciton has the following form:

$$
\begin{aligned}
H_{e x}\left(\vec{r}_{e}, \vec{r}_{h}\right)= & E_{G}+H_{e l}\left(\vec{r}_{e}\right)+H_{h}\left(\vec{r}_{h}\right) \\
& +V_{\text {coul }}\left(\vec{r}_{e}, \vec{r}_{h}\right)+V\left(\vec{r}_{e}, \vec{r}_{h}\right)+2 E_{\text {self }},
\end{aligned}
$$

where, in addition to the usual Coulomb attraction potential between electron and hole, $V_{\text {coul }}\left(\vec{r}_{e}, \vec{r}_{h}\right)=e^{2} / 4 \pi \epsilon_{0} \epsilon_{a}\left|\vec{r}_{e}-\vec{r}_{h}\right|$, we have added two additional contributions: the position dependent term,

$$
\begin{aligned}
V\left(\vec{r}_{e}, \vec{r}_{h}\right)= & -\frac{e^{2}}{4 \pi \epsilon_{0} \epsilon_{a}} \frac{2}{\pi}\left(\frac{\epsilon_{a}}{\epsilon_{b}}-1\right) \sum_{m=-\infty}^{+\infty} \int_{0}^{\infty} C_{m}\left(k R, \frac{\epsilon_{a}}{\epsilon_{b}}\right) \\
& \times \cos \left[k\left(z_{e}-z_{h}\right)\right] \cos \left[m\left(\theta_{e}-\theta_{h}\right)\right] I_{m}\left(k \rho_{e}\right) I_{m}\left(k \rho_{h}\right),
\end{aligned}
$$

and the position independent term called self-energy $E_{\text {self. }}$. In Eq. (8), the symbol 


$$
C_{m}\left(k R, \frac{\epsilon_{a}}{\epsilon_{b}}\right)=\frac{K_{m}(k R) K_{m}^{\prime}(k R)}{I_{m}(k R) K_{m}^{\prime}(k R)-\frac{\epsilon_{a}}{\epsilon_{b}} I_{m}^{\prime}(k R) K_{m}(k R)},
$$

and $\left(I_{m}, I_{m}^{\prime}\right)$ are modified Bessel functions of the first kind and its derivative and $\left(K_{m}, K_{m}^{\prime}\right)$ are modified Bessel functions of the third kind and its derivative. Detailed analysis of these potential components can be found in Ref. 11. In the latter reference, the authors constructed an analytical form for $E_{\text {self }}$ which is applicable to a wide class of semiconductors. In our paper, we borrow their expression: $E_{\text {self }}(R)=e^{2} /\left(4 \pi \epsilon_{0} \epsilon_{a} R\right)$ $\times 0.89 \times\left[\left(\epsilon_{a} / \epsilon_{b}\right)^{0.59}-1\right]$.

We minimized the Hamiltonian $H_{e x}$ over a class of variational wave functions $\Psi_{e x}: E_{t o t}=\min _{\Psi}\left\langle\Psi_{e x}\left|H_{e x}\right| \Psi_{e x}\right\rangle$. The $\nu$ component of the exciton trial wave function $\Psi_{e x}$ with zero center of mass momentum was assumed to be

$$
\begin{aligned}
\Psi_{e x}^{\nu}= & \sum_{j=0}^{J-1} \sum_{m=-M}^{M} \sum_{l=0}^{L-1} C_{j, m, l}^{\nu} \phi_{j}\left(z_{e}-z_{h}\right) \frac{1}{\sqrt{2 \pi}} \frac{\sqrt{2}}{R J_{1}\left(\alpha_{01}\right)} \\
& \times J_{0}\left(\alpha_{01} \frac{r_{e}}{R}\right) \frac{e^{i m \phi_{h}}}{\sqrt{2 \pi}} \frac{\sqrt{2}}{R J_{m+1}\left(\alpha_{m l}\right)} J_{m}\left(\alpha_{m l} \frac{r_{h}}{R}\right),
\end{aligned}
$$

where

$$
\phi_{j}(z)=e^{-a^{2} z^{2} / 2} \frac{\sqrt{a}}{\sqrt{\sqrt{\pi} 2^{j} j !}} H_{j}(a z)
$$

is a normalized eigenfunction of the harmonic oscillator with scaling parameter $a$. In our simulations, we used $J=5, L=4$, and $M=4$. We performed minimalization of the exciton ground state energy with respect to the scaling parameter $a$ as well as the linear parameters $C_{j, m, l}$. In the exciton trial function, we included only one electron subband $(m=0, l$ $=0$ ) because, as will be seen in the next section, the quantum
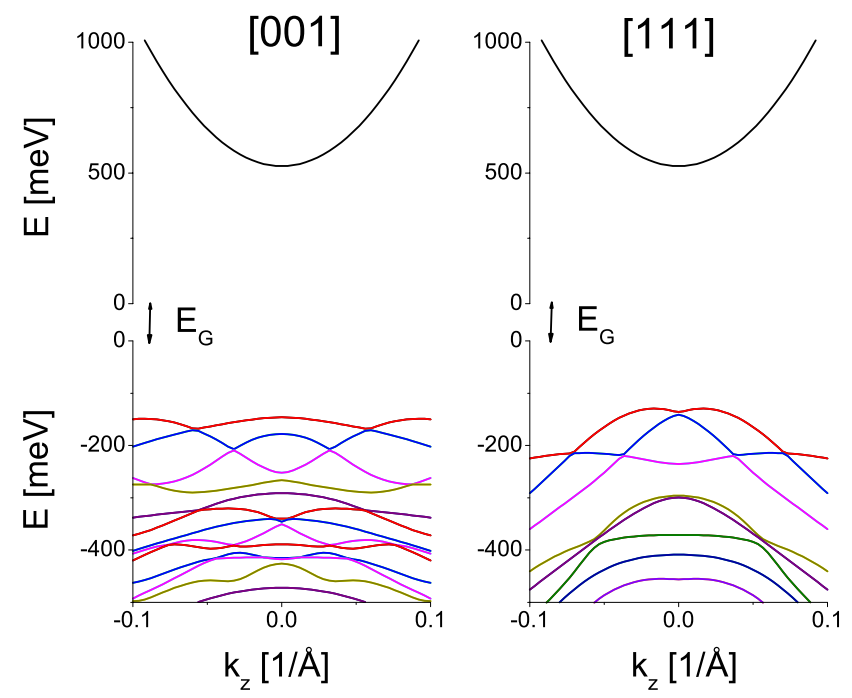

FIG. 2. (Color online) Typical dispersion relation for the conduction and the valence bands for a $R=25 \AA$ nanowire for two different growth directions. All points are twofold degenerate with respect to the spin degree of freedom. confinement effects are much stronger for the conduction band than for the valence band.

\section{RESULTS AND DISCUSSION}

\section{A. One-particle states}

For our numerical calculations, we used the material parameters of GaAs with electron mass $m_{e}=0.067 m_{0}$, Luttinger parameters: $\gamma_{1}=7.65, \gamma_{2}=2.41$, and $\gamma_{3}=3.28$, and dielectric constant $\epsilon_{a}=12.56$ and we assumed that the nanowire is surrounded by vacuum, i.e., $\epsilon_{b}=1$.

We consider two orientations of the crystal lattice with respect to the cylindrical coordinates of the infinite wire: the [001] and [111] configurations. First, we show results for the [001] configuration and than for the [111] configuration.

\section{1. [001] orientation}

In Fig. 2, we show a typical energy spectrum of the conduction and valance bands in the [001] and the [111] configurations. We took for the diameter of the wire $D=50 \AA$ which is smaller than the Bohr radius of bulk GaAs $\left(a_{B}\right.$ $=120 \AA$ ).

The smaller electronic mass in the conduction band results in only one electron branch in the energy range of $500 \mathrm{meV}$ that will contribute. On the other hand, for the valence band in the same energy range, we have many

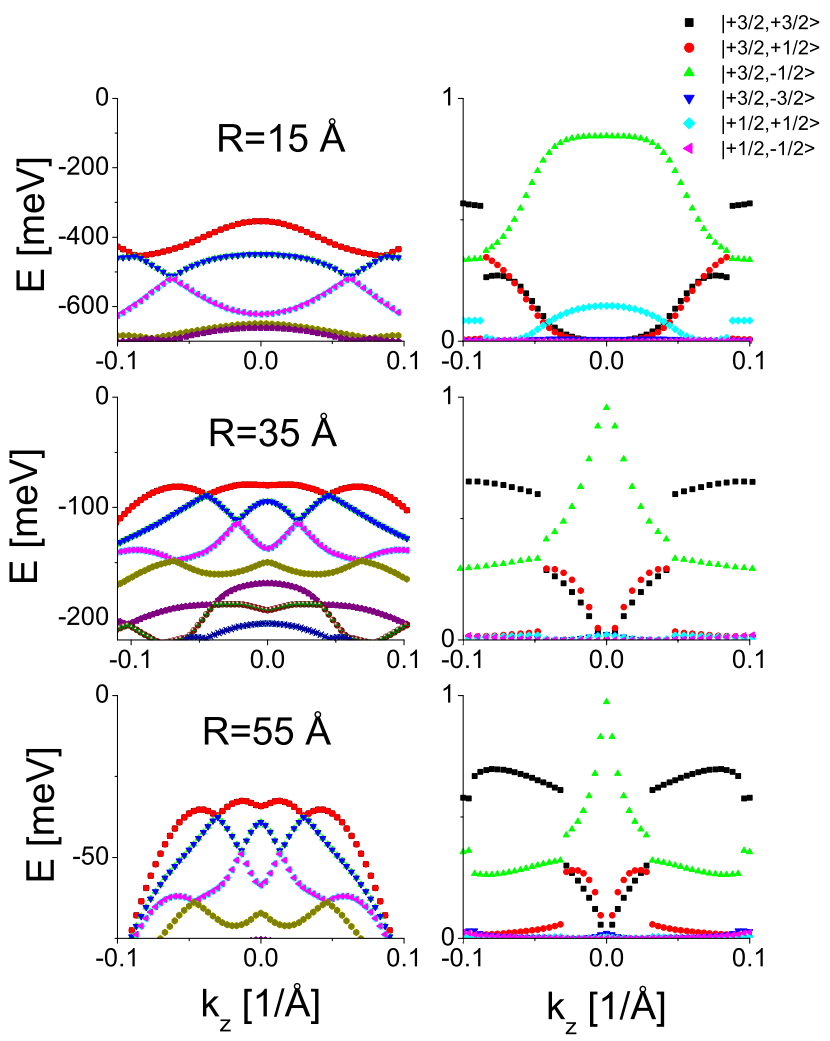

FIG. 3. (Color online) Dispersion of the valence band in the [001] configuration for three radii $R=15,35$, and $55 \AA$. To the right of each dispersion we plot the spinor distribution of the ground state. 


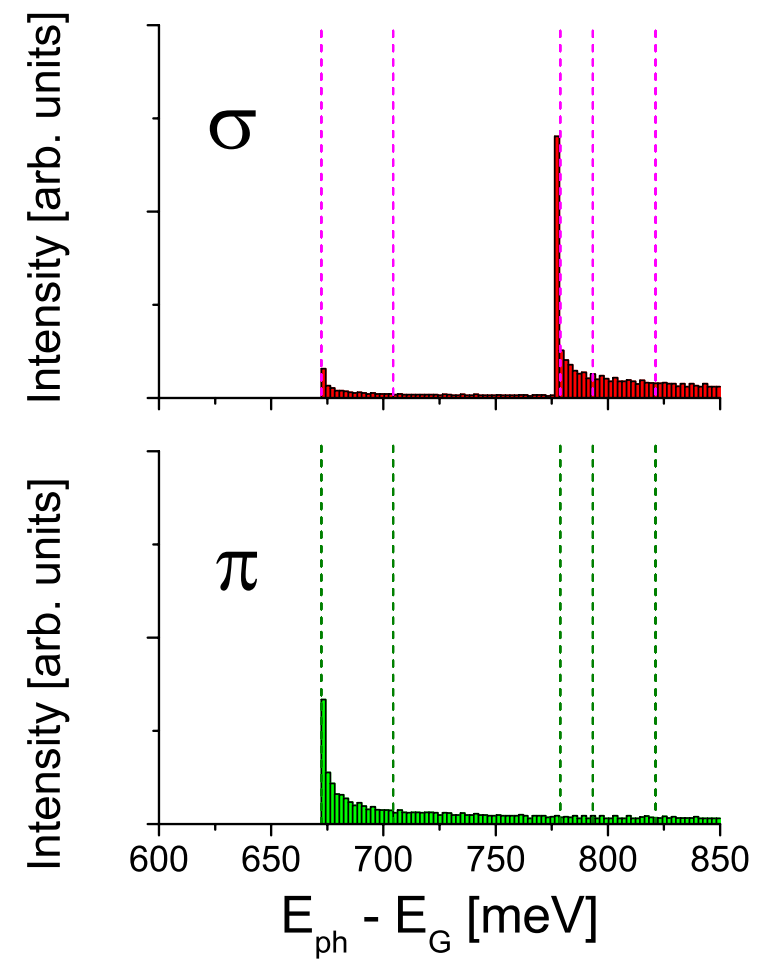

FIG. 4. (Color online) Free electron absorption spectrum for the [001] configuration at $T=0 \mathrm{~K}$ for $R=25 \AA$. Thin vertical dashed lines indicate energy separation between conduction band states and valence band states at $k_{z}=0$.

branches contributing due to the larger effective mass. These observations are useful to construct the exciton wave function. From Fig. 2, the confinement energy of the electron is about $500 \mathrm{meV}$ but for the hole it is only about $150 \mathrm{meV}$. An unusual characteristic of these quasi-one-dimensional systems is that there are multimaxima in the energy spectrum of the ground state of the hole (i.e., a "camel back" structure). This nonmonotonic dispersion is due to the interaction between the light and the heavy hole states.

In Fig. 3, we show the evolution of the valence band spectrum as a function of the radius of the wire for $R=15$, 35 , and $55 \AA$. For each spectrum, we plot the content of the spinor $\nu$ component to the corresponding ground state: 0 $<P_{\nu}\left(k_{z}\right)=\int\left|\Psi_{e x}^{\nu}\left(\rho, k_{z}\right)\right|^{2} d^{2} \rho<1$. In Fig. 3, we can see clearly the camel back structure that shrinks when the radius of the wire increases. Second, below $R \approx 35 \AA$, there are only three maxima but for larger $R$, we find four local maxima. Our calculations show also that there are energy crossings at some finite $k_{z}$ where simultaneously the spin state of the hole is changing. For example, for $R=35 \AA$, the spin state of the ground state at $k_{z} \approx 0.5$ is changing from $-1 / 2$ to $+3 / 2$. Around $k_{z}=0$, the main contribution to the ground state is made by the light component of the wave function: $|+3 / 2, \pm 1 / 2\rangle$. The region of domination of light-hole component decreases when the diameter of the wire increases. Contribution of the spin off band is minimal but increases with decreasing diameter of the wire.

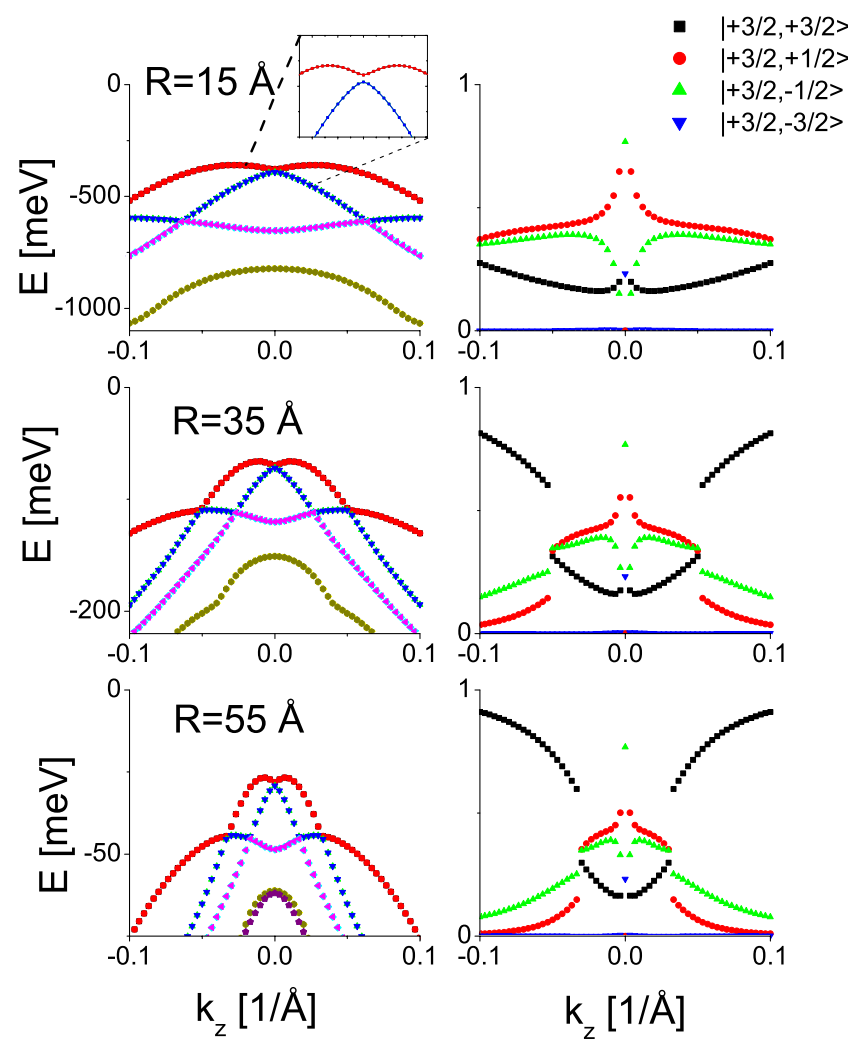

FIG. 5. (Color online) The same as Fig. 3 but now for a wire grown along the [111] crystallographic direction.

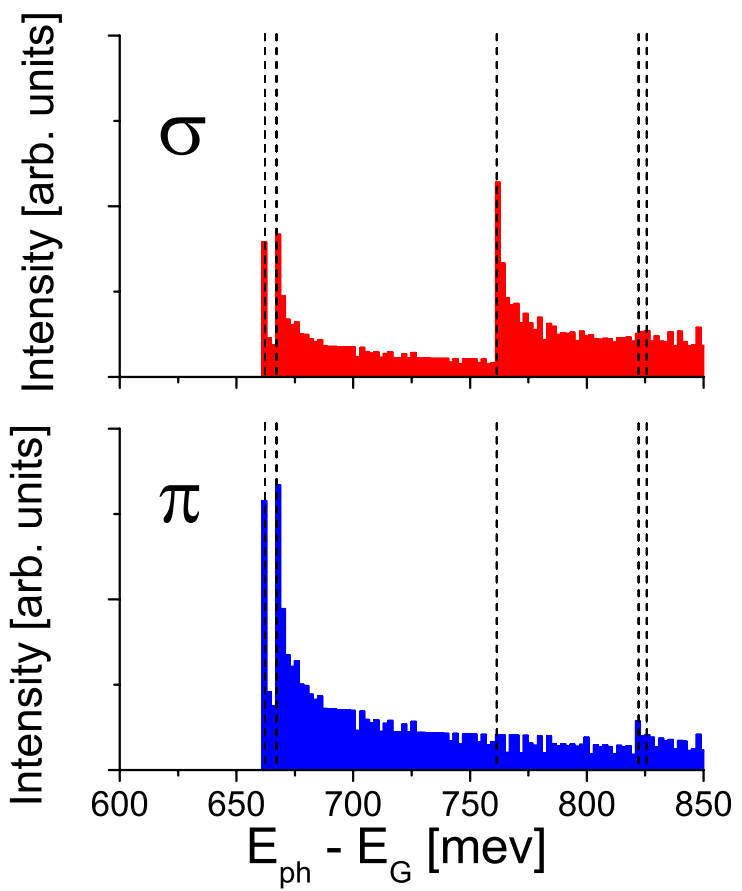

FIG. 6. (Color online) Free electron absorption spectrum for the [111] configuration at $T=0 \mathrm{~K}$ and $R=25 \AA$. Vertical lines indicate the energy separation between the conduction and the valence band state at $k_{z}=0$. 

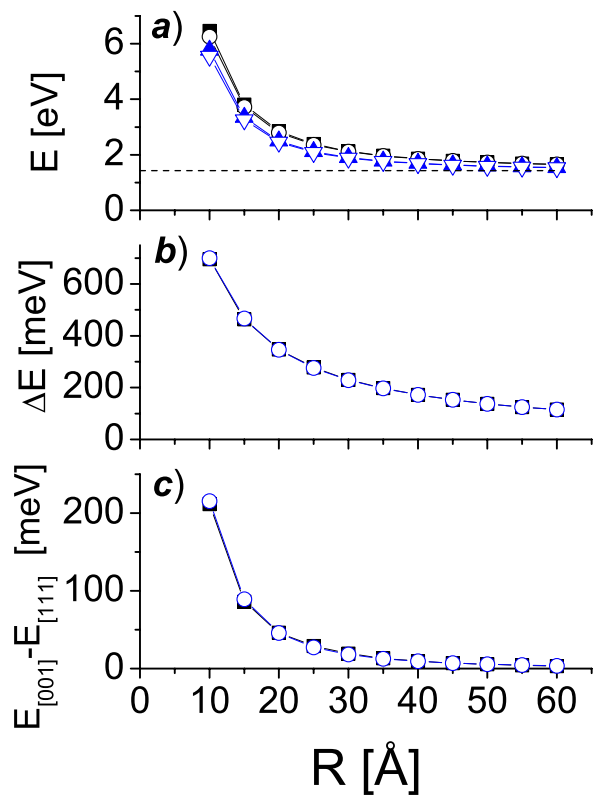

FIG. 7. (Color online) (a) Exciton PL transition energies $E_{[001]}$ (solid squares and solid up triangles) and $E_{[111]}$ (opened circles and open down triangles) as a function of radius $R$. Results for bare Coulomb potential are marked with solid up and open down triangles. Results with Coulomb potential plus dielectric mismatch are marked with solid squares and open circles. Dashed line indicates energy gap of bulk GaAs crystal assumed to be $E_{G}=1430 \mathrm{meV}$. (b) The energy difference $\Delta E$ in the transition energy resulting from the dielectric mismatch effect only. Growth direction does not influence this difference and $\Delta E \sim R^{-1}$ is almost the same for the [001] and [111] growth directions. (c) The difference $E_{[001]}-E_{[111]}$ in the case when only the bare Coulomb potential is taken into account (open circles) and when additionally the dielectric mismatch is included (solid squares). $E_{[001]}-E_{[111]}$ is well approximated by the dependance $\sim R^{-2.2}$.

In Fig. 4, we show the optical absorption spectrum for a $R=25 \AA$ nanowire. Vertical lines indicate transition energies between conduction and valence bands at $k_{z}=0$. Absorption spectrum indicates quasi-one-dimensional behavior with absorption intensity $I \approx \Theta\left(E-E_{i f}\right) / \sqrt{E-E_{i f}}$. We see that not all "main" transitions produce peaks as a consequence of selection rules (forbidden transitions). Our results indicate also that the lower energy absorption peak is larger in magnitude in the $\pi$ polarization than in $\sigma$ polarization. Second, we expect that in the $\pi$ polarization, the higher energy peak appears roughly $200 \mathrm{meV}$ above the first one. The higher energy peak has a larger intensity due to selection rules.

\section{2. [111] orientation}

We used our numerical procedure to investigate the electronic properties of a nanowire grown along the [111] crystallographic direction in order to investigate the dependence on the growth direction. Electron spectrum and electron wave functions are identical to the [001] configuration and will not be discussed.

In Fig. 5, we show the dispersion and the distribution of the spinor components of the wave function of the hole. As
TABLE I. Numerical values of the effective masses in the direction of the wire (subscript 3 ) and in the plane of the wire (subscript $\|$ ) for the [001] and the [111] orientations. The following Luttinger parameters for the bulk GaAs semiconductor were used: $\gamma_{1}=7.65, \gamma_{2}=2.41$, and $\gamma_{3}=3.28$. We give also the numerical value of the reduced mass $\mu$ of the quasi-one-dimensional exciton.

\begin{tabular}{llll}
\hline \hline HH $\left(m_{0}\right)$ & $m_{\|}=\frac{1}{\gamma_{1}+\gamma_{2}}=0.10$ & $>$ & $m_{\|}=\frac{1}{\gamma_{1}+\gamma_{3}}=0.09$ \\
& $m_{3}=\frac{1}{\gamma_{1}-2 \gamma_{2}}=0.35$ & $<$ & $m_{3}=\frac{1}{\gamma_{1}-2 \gamma_{3}}=0.92$ \\
LH $\left(m_{0}\right)$ & $m_{\|}=\frac{1}{\gamma_{1}-\gamma_{2}}=0.19$ & $<$ & $m_{\|}=\frac{1}{\gamma_{1}-\gamma_{3}}=0.23$ \\
& $m_{3}=\frac{1}{\gamma_{1}+2 \gamma_{2}}=0.08$ & $>$ & $m_{3}=\frac{1}{\gamma_{1}+2 \gamma_{3}}=0.07$ \\
0.036 & $>$ & 0.034 \\
\hline \hline
\end{tabular}

compared to the results of the [001] configuration (cf. Fig. 3 ), there are several differences between the characteristics of both nanowires. First, for the [111] case, there is a minigap at the $\Gamma$ point between the two highest lying states. In Fig. 5, this is not clearly seen but it is seen in the absorption spectrum (see Fig. 6). A minigap is decreasing function of $R$; for $R=15 \AA$, it is about $16 \mathrm{meV}$, but for $R=50 \AA$, it is less than $1 \mathrm{meV}$. Second, the distribution of the spinor components is different as compared to the [001] case, but around $k_{z}=0$, the dominant contribution to the ground state comes from the light-hole subband.

In Fig. 6, we show the absorption spectrum for a $R$ $=25 \AA$, nanowire in the [111] configuration. We performed calculations for two polarizations of light. In $\sigma$ and $\pi$ polarizations, above $650 \mathrm{meV}$, there are two peaks which indicate a minigap in the spectrum at the $\Gamma$ point. Due to selection rules, our results indicate that absorption is larger in $\pi$ polarization than in $\sigma$ polarization. Additionally, second stronger peak appears in the $\sigma$ polarization at higher energy (200 meV).

\section{B. Quasi-one-dimensional exciton}

Now, we present calculations of exciton states including the dielectric mismatch contribution to the interaction between the charged particles. As was shown previously, this effect greatly increases the Coulomb interaction. ${ }^{11,23}$

Top panel of Fig. 7 shows the ground state photoluminescence (PL) energy in the [001] (line with solid squares) and [111] (line with open circles) configurations as a function of radius $R$. PL energy includes confinement energies as well as self-energies of the electron and the hole. Dashed line indicates the energy gap of the bulk material. We found that in the range of $R=1-6 \mathrm{~nm}$, the PL energy is well approximated by $\sim R^{-p}$ with $p \approx 1.8$ for both orientations of the crystal.

The PL energy in both configurations is not the same. To explain this difference $\left(E_{[001]}-E_{[111]}\right)$, we calculated the "effective masses" coming from the diagonal components in the KL. Table I gives the numerical values of these "masses." 
Because spatial quantization of the system is in the plane perpendicular to the spin quantization, so the ground state has a large light-hole contribution. From Table I, we also can see that the "perpendicular" effective mass $\left(m_{\|}\right)$of the light hole is smaller for the [001] case than for the [111] case, $m_{\|}=0.19 m_{0}$ and $m_{\|}=0.23 m_{0}$, respectively. We would like to stress that for other compounds, there might be different mathematic relations (middle columns on Table I) between masses for the two growth directions. The difference of $m_{\|}$ causes different spatial quantizations in the plane and so causes a difference in the PL energies for the [001] and [111] configurations. Line with black squares in Fig. 7(c) shows the difference $E_{[001]}-E_{[111]}$ which can be as large as tens of $\mathrm{meV}$ for $R<3 \mathrm{~nm}$.

For comparative reasons we also performed a calculation of the exciton PL energy using only the bare Coulomb interaction between the charged particles. This type of calculation might be relevant for a core-shell nanowire with a thick shell and high barriers. High barriers are needed because in our model we assume localization of electronic charge in the wire. In Fig. 7(a), we present the PL energies for a nanowire grown along the [001] (line with solid up triangles) and [111] (line with open down triangles) crystallographic directions. In the range of $R=1-6 \mathrm{~nm}$, the PL energy can well be approximated by the function $\sim R^{-p}$ with $p \approx 2.0$ for both orientations of the crystal. This value of $p$ is slightly larger than for the case when the dielectric mismatch effect is included. The energy difference $E_{[001]}-E_{[111]}$ is shown in Fig. 7(c) (open circles). As can be seen, $E_{[001]}-E_{[111]}$ is practically the same for the bare Coulomb interaction (solid squares) and Coulomb interaction which is adjusted with dielectric mismatch effect (open circles). Using fitting function $R^{-p}$ to these curves in the domain $R=1-6 \mathrm{~nm}$, we obtained $p=2.2$.
As seen from Fig. 7(a), the dielectric mismatch effect increases the PL energy. The difference may be as large as $0.7 \mathrm{eV}$ for small $R$ but it decreases with increasing radius of the wire. For $R=6 \mathrm{~nm}$, we found that the difference is about $0.1 \mathrm{eV}$. In Fig. 7(b), we plot the energy difference $\Delta E$ coming from the dielectric mismatch of the system for the [001] (solid squares) and [111] (open circles) growth directions. It is apparent that the growth direction does not have any effect on $\Delta E$. We checked that $\Delta E \sim R^{-p}$ with $p=1$ is a very good approximation in the range of $R=1-6 \mathrm{~nm}$.

\section{CONCLUSIONS AND SUMMARY}

We performed a numerical calculation of the energy spectrum, the absorption spectrum, and the PL transition energy of excitons for a free-standing nanowire. We used the $\mathbf{k} \cdot \mathbf{p}$ model to describe direct wide gap semiconducting nanowires. Numerical results were presented for GaAs but our model is also applicable for II-VI compounds such as CdTe. We investigated cylindrical nanowires with radius up to $6 \mathrm{~nm}$ grown along the [001] and [111] directions. We showed that, in both cases, quantization of the system and heavylight hole interaction causes an unusual energy dispersion and absorption spectrum of nanowire, i.e., a camel back structure of the energy dispersion and a minigap in the absorption spectrum. We showed also that the photoluminescence energy of the exciton depends on the growth direction of the nanowire.

\section{ACKNOWLEDGMENTS}

This work is supported by the European Network of Excellence: SANDiE, the Belgian Science Policy (IAP), and the Flemish Science Foundation (FWO-Vl).

\footnotetext{
*pawel.redlinski@ua.ac.be

francois.peeters@ua.ac.be

${ }^{1}$ R. S. Wagner and W. C. Ellis, Appl. Phys. Lett. 4, 89 (1964).

${ }^{2}$ Z. H. Wu, X. Y. Mei, D. Kim, M. Blumin, and H. E. Ruda, Appl. Phys. Lett. 81, 5177 (2002).

${ }^{3}$ A. M. Morales and C. M. Lieber, Science 279, 208 (1998).

${ }^{4}$ H.-Y. Li, O. Wunnicke, M. T. Borgstrom, W. G. G. Immink, M. H. M. van Weert, M. A. Verheijen, and E. P. A. M. Bakkers, Nano Lett. 7, 1144 (2007).

${ }^{5}$ K. Haraguchi, T. Katsuyama, K. Hiruma, and K. Ogawa, Appl. Phys. Lett. 60, 745 (1992).

${ }^{6}$ X. Duan, J. Wang, and C. M. Lieber, Appl. Phys. Lett. 76, 1116 (2000).

${ }^{7}$ D. Karanth and H. Fu, Phys. Rev. B 74, 155312 (2006).

${ }^{8}$ A. Shabaev and A. L. Efros, Nano Lett. 4, 1821 (2004).

${ }^{9}$ Y. M. Niquet, Nano Lett. 7, 1105 (2007).

${ }^{10}$ T. B. Hoang, L. V. Titova, J. M. Yarrison-Rice, H. E. Jackson, A. O. Govorov, Y. Kim, H. J. Joyce, H. H. Tan, C. Jagadish, and L. M. Smith, Nano Lett. 7, 588 (2007).

${ }^{11}$ A. F. Slachmuylders, B. Partoens, W. Magnus, and F. M. Peeters,
}

Phys. Rev. B 74, 235321 (2006).

${ }^{12}$ Y. M. Niquet, A. Lherbier, N. H. Quang, M. V. Fernandez-Serra, X. Blase, and C. Delerue, Phys. Rev. B 73, 165319 (2006).

${ }^{13}$ M. H. Huang, S. Mao, H. Feick, H. Yan, Y. Wu, H. Kind, E. Weber, R. Russo, and P. Yang, Science 292, 1897 (2001).

${ }^{14}$ J. Wang, M. S. Gudiksen, X. Duan, Y. Cui, and C. M. Lieber, Science 293, 1455 (2001).

${ }^{15}$ Y. Cui, Z. Zhong, D. Wang, W. U. Wang, and C. M. Lieber, Nano Lett. 3, 149 (2003).

${ }^{16}$ N. Malkova and C. Z. Ning, Phys. Rev. B 74, 155308 (2006).

${ }^{17}$ J. M. Luttinger and W. Kohn, Phys. Rev. 97, 869 (1955).

${ }^{18}$ T. Dietl, H. Ohno, and F. Matsukura, Phys. Rev. B 63, 195205 (2001).

${ }^{19}$ A. Baldereschi and N. O. Lipari, Phys. Rev. B 8, 2697 (1973).

${ }^{20}$ G. Fishman, Phys. Rev. B 52, 11132 (1995).

${ }^{21}$ J.-B. Xia, Phys. Rev. B 43, 9856 (1991).

${ }^{22}$ P. Y. Yu and M. Cardona, Fundamentals of Semiconductors, 3rd ed. (Springer, New York, 2003), p. 260.

${ }^{23}$ L. Banyai, I. Galbraith, C. Ell, and H. Haug, Phys. Rev. B 36, 6099 (1987). 